\title{
Performance Analysis of Avalanche Photodiode for Direct Detection LADAR Receivers
}

\author{
تحليل أداء الثنائى الضوئى الانهيارى لمسنقبلات الرادارات الليزرية ذات الكشف المباشر \\ M. B. EL MASHADE, H. Konber, M. Ashour \& A. AboElEzz \\ Electrical Engineering Dept., Faculty of Engineering, Al_Azhar University, \\ Nasr City, Cairo, Egypt \\ ElMashade@yahoo.com
}

\begin{abstract}
ملخص:

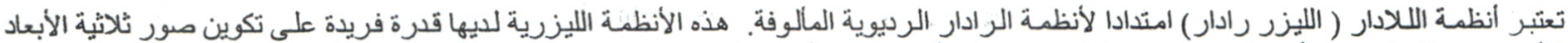

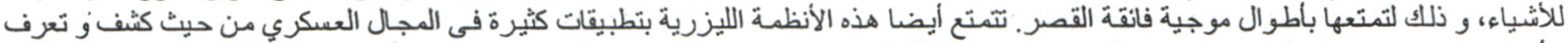

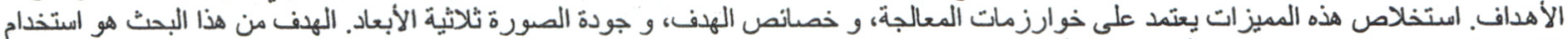

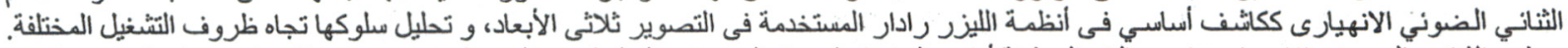

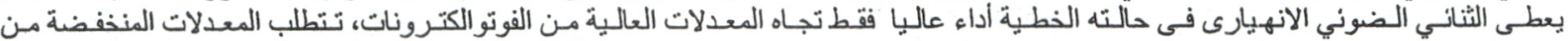

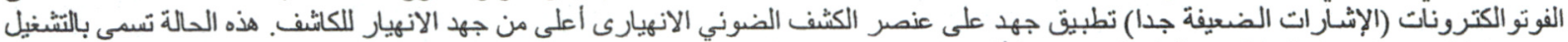

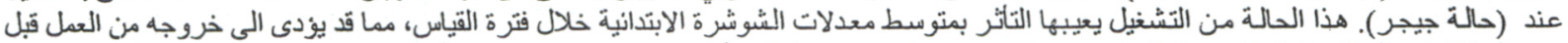

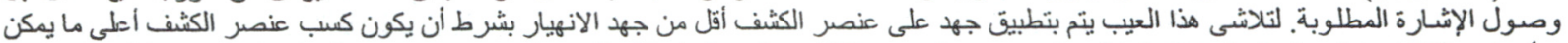

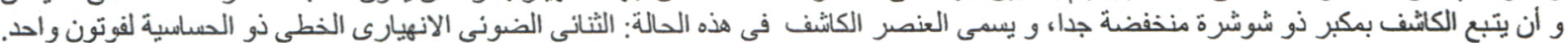
هذه الحالة تميز بتغلبها على عيوب حالة جيجر . تمت دراسة احتمالات الكشف و احتمالات الإنذارات الخاطنة لكل حالة تشغيل.
\end{abstract}

\section{$\underline{\text { Abstract }}$}

LADAR systems constitute a direct extension of the conventional radar techniques. Because they operate at much shorter wavelengths, LADARs have the unique capability to generate 3D images of objects. These laser systems have many applications in the defense gelds concerning target detection and identification. The extraction of these features depends on the processing algorithms, target properties and 3D images quality. Our scope in this research is to use the Avalanche photodiode as a basic detector in three dimensional imaging LADAR systems and analyze its behavior against different operating conditions. Avalanche photodiode in its linear mode gives good performance only for high count rate of photo-electrons. In the case of low count rates (extremely weak signals), this device must be biased above its breakdown voltage in order to have higher sensitivity. This situation is known in the literature as Geiger-mode operation. This mode of operation suffers from mean primary noise rates in the measured interval. This in turn may cause the detector not respond before receiving the desired signal. When the Avalanche photodiode is biased below its breakdown voltage with highest achievable gain value, and followed by an ultra low noise amplifier, it becomes sensitive to the single photo-electron. This operating mode is called Linear-mode single photon. The operation of the detector in this mode overcomes the drawbacks of the Geiger-mode. Detection and false alarm probabilities are analyzed for each one of these operating situations.

Keywords: Avalanche photodiode, Three-Dimensional Imaging, Direct detection, LADAR

\section{Introduction}

LADARs or laser radars, detect targets using a laser source for producing the electromagnetic radiations. The prevalent method is to send a pulse and measure its time of flight when it arrives back at the receiver. The measured time gives an estimation of the target range for each laser shot. Such LADAR systems provide distant images and could be used as target seekers. Avalanche photodiodes (APDs), on the other hand, are commonly used in light wave systems as detectors of optical signals because of their multiplication property which can enhance the signal-to-noise ratio (SNR) of a detected optical signal, even though APD's introduce excess

Accepted November 14, 2011. 


\section{E. 54 M. B. El-Mashade, H. Konber, M. Ashour and A. Abo-El-Ezz}

noise. In addition, they represent an important part of optical devices in fiber-optic communication systems. As a key component in these transmission systems, ultrahigh-speed photodetectors are able to not only increase the transmission capability in a single channel, but also reduce the required number of channels and complexity of management in a wavelength-division-multiplexing (WDM) fiber communication system [11]. They operate by converting each detected photon into a cascade of electrons. Therefore, a pulse of light can produce a sufficiently high charge to be readily detected by the electronics following the APD. Moreover, to achieve high bandwidth-efficiency product performance, avalanche photodiodes (APDs) are the preferred type of optical detectors due to their internal gain and high sensitivity in comparison with other types of photodiodes. These detectors can be operated in one of the following three modes: linear mode, Geiger-mode photon counting (GM-APD), and Linear mode single photon counting (LM-SPAPD). Owing to the afterpulse phenomenon, Geiger mode suffers from long reset time following each detection event $[5,6]$. The very large breakdown current that flows in a Geiger APD during a detection event populates traps that release their trapped carriers over time." Once the Geiger APD returns to service after the firing process, the more likely it is to trigger-off of a carrier that was trapped during the previous detection event, registering a spurious count. Quench times greater than $1 \mu$ s are generally necessary, and as the operating temperature becomes lower, as the APD requires longer time for the quench duration. In contrast, the current that flows in a linearmode APD is too small to fill any appreciable population of traps, so linear APDs return from detection events as soon as the current pulse clears the diode junction (typically in $\approx 1 \mathrm{~ns}$ ). A brief discussion about the behavior of these modes will be given here.

An APD is affected by two sources of noise, dark noise and afterpulsing. Dark noise is due to the thermal excitation of electrons in the ionization region. The dark noise is reduced by adjusting the temperature and the reverse bias, which reduce also the quantum efficiency (QE). Afterpulsing is due to the trapping of electrons during an avalanche in defect levels in the ionization area of the APD. The trapped charges trigger an avalanche during the next gate. An easy way to reduce the afterpulsing is to use a dead time longer than the lifetime of the trapped carriers, which is of the order of several microseconds.

In the first mode of operation, the APD is biased below its breakdown voltage [1] in such a way that the average output current is proportional to the incident optical power and its magnitude is proportional to the primary photocurrent with the internal avalanche gain as a constant of proportionality. It is of importance to note that the performance of photodetector in this mode of operation is limited by the APD excess noise which is excited by the randomness of the APD gain mechanism. On the other hand, the second mode of operation is characterized by biasing the APP above its breakdown voltage with an amount $\Delta v$ and the voltage remains in this new value until a generation of a primary electronhole pair, either thermally or optically, which in turn initiate the breakdown. One of the generated carriers enters the multiplication region of the depletion layer to initiate the discharge. This behavior will lead to a strong output current spike that can be counted. So, Geigermode operation is a nonlinear digital detection approach and so, it cannot resolve multiple photons. In the last one of modes, [2] the APD is biased within one or two volts before the breakdown voltage takes place. The average gain in such a photon counter should be set to its highest stable value and this necessitates a temperature control as well as a highly stable voltage supply for the APD to be satisfactory operated. It is well known that each photon absorbed in this mode of operation will result in a photocurrent pulse and the amplitude of this pulse is much smaller than that obtained by (GM-APDs). Due to the modest value of the generated pulse, the APD can not be used individually; instead it is used in conjunction with an ultra low-noise preamplifier and a comparator with a threshold of 5-6 times greater than the amplifier noise level [2]. The result of this arrangement is that the APD becomes capable of counting those pulses for which the gain exceeds the threshold level. The major advantage of this type of photon counting is its ability of eliminating both after pulsing and slow recovery process following each avalanche breakdown that may be observed in the case of GM operation. As a result of this behavior, the counter dead time is limited only by the electrical bandwidth of the APD and its subsequent circuits.

Our scope in this manuscript is to evaluate the performance of such LADAR systems that employ the APD as a photodetector. The primary concern here is to determine the false alarm and detection probabilities of the underlined scheme in each one of its modes of operation.

Detection and false alarm probabilities in the presence of dark current and background light have been calculated for linear mode operation [3], and Geiger mode operation [4]. Comparison has been presented between these two modes of operations [5, 6]. Our study takes into account the previous results and introduces a complete view for the three types of APD operation applied to LADAR system model. The organization of 
the manuscript is as follows: section 2 describes the statistical model of the signal being detected. Section 3 discusses the statistics of the APD in linear mode operation with the performance plots. Section 4 depicts the statistic of the APD in Geiger-mode operation. Section 5 displays the statistics the APD in linear mode single photon operation. Section 6 discusses the performance plots for Geiger-mode operation and linear mode single photon operation for single and multiple pulses. Our conclusions are summarized in section 7.

\section{Statistical Model Description}

\subsection{Proposed System description}

The proposed direct detection LADAR system (i.e., allowing range measurements only) based APD focal plane array (FPA) system is as follow [3, 4, 5, 6, 9]; APD FPA with $256 \times 256$ pixel array size, average fill factor $\gamma=70 \%$, aperture diameter $d=0.2 \mathrm{~m}$, optics with focal number $f / 2$, and angular pixel pitch of $\phi_{\text {pixel }}=0.1$ $m$ rad. The angular pixel pitch and the pixel intermediate field of view (IFOV) are not equal as the active pixel size is less than the distance between the pixels. Each detector have quantum efficiency $\eta=0.38$. Assumed laser source is with photon wavelength of $\lambda=1064 \mathrm{~nm}$ (i.e., Nd Yag laser). The beam divergence of the illuminating laser is given by: $\theta=\sqrt{2} \times 256 \times \phi_{\text {pixel }} \approx 36.2 \mathrm{~m} \mathrm{rad}$, that illuminate the field of the whole array. The detector is activated for a period of time corresponding to range gate of interest called gate time $\tau_{\text {gate }}$, that time is divided to the smallest subdivision of time equal to the pulse duration (i.e., measurement time $\tau$ ) and therefore the smallest distance resolution $\Delta r$ that the LADAR system can record. The measurement gate will assumed to be $200 \mathrm{~m}$ starting from $r_{s}=500 \mathrm{~m}$, with range resolution of $\Delta r=0.2 \mathrm{~m}$, that required integration time of $\tau=2 \Delta r / c \approx 1.33 \mathrm{~ns}$, where $c$ denotes the velocity of light in free space. The range to the target $r$ is related to target range bin $B$ as $r=r_{s}+B \cdot \Delta r$.

\subsection{Signal Model}

When a diffused target is illuminated by a LADAR, the mean number of signal photons per time interval reflected from that target is a random process. It is shown that the target signal creates $k$ primary photo-electrons in the target bin, the distribution of which follows the negative-binomial formula [7] which is given by;

$$
P_{s}(k)=\frac{\Gamma(k+M)}{k ! \Gamma(M)}\left(1+\frac{M}{\bar{N} s}\right)^{-k}\left(1+\frac{\bar{N} s}{M}\right)^{-M t}
$$

In the above expression, $P_{S}(k)$ represent the probability density function (PDF) of the $k^{\text {th }}$ primary photo-electrons, $\overline{N_{s}}=\langle k\rangle$ is the mean number of photo-electrons in the measured interval, $M$ denotes the number of degrees of freedom in the sampled intensity (speckle) distribution, and $\Gamma(\mathrm{x})$ designs the gamma function. It is worth noted that $M$ may be non-integer in the case where it is determined at the receiving aperture.

The background photons and dark current count statistics are generally obeying Poisson distribution since they have nearly constant parameter rates. It is noted that a Poisson process has the reproductive property which means that the sum of any number of statistically independent Poisson-distributed random variables is itself a Poisson distributed random variable, with a mean rate given by the sum of the individual mean rates. Additionally, the number of events generated in any time interval is statistically independent of the number generated in any other non-overlapping interval.
After this brief introduction, let us now turn our attention to the problem of detection of the reflected laser light. It is more reasonable to assume that it follows a Poisson process given that the following two conditions are fulfilled; The first condition is that the quantity $M / \bar{N}$ must be much greater than one, while the second one concerns with the atmospheric turbulence that must have a negligible effect on the speckle pattern at the receiving end. For the last condition to be satisfied, the propagation distance must be short vertical (in our analysis, the maximum measurement distance is $700 \mathrm{~m}$ ).

\subsection{Calculation of main signal and background radiation}

This section is devoted to derive the basic formulas for the mean number of photo-electrons generated by reflected laser pulse as a function of target range, and that caused by natural background sunlight reflection. 


\section{E. 56 M. B. El-Mashade, H. Konber, M. Ashour and A. Abo-El-Ezz}

The mean number of primary photoelectrons per pixel generated by a collection of laser photons reflected from resolved target (i.e., collected light from an area

$$
\begin{aligned}
\bar{N}_{s} & =\frac{E_{1}}{h v} \cdot \eta \cdot \rho \cdot \frac{A_{\text {IFov }}}{A_{\text {spot }}} \cdot \frac{A_{R}}{\pi r^{2}} T^{2} \\
& =\frac{E_{1} \lambda}{h c} \cdot \eta \cdot \rho \cdot \gamma \cdot\left(\frac{\phi_{\text {pixel }}}{\theta}\right)^{2} \exp \left(-\frac{2 r}{V}\right) \frac{d^{2}}{4 r^{2}}
\end{aligned}
$$

$A_{\text {HKOW }}$ on the target $\leq$ target cross section) at range $r$ is calculated from laser radar equation as [5];
In the above expression, $E$, represents the transmitted laser pulse energy, $\lambda$ is laser wavelength, $r$ is range to the target with reflectivity $\rho$. Meteorological visibility $V$ determines the propagation loss $T$ through the atmpsphere, $\phi_{\text {pixel }}$ denotes the angular pixel pitch, $\theta$ is the laser divergence to illuminate the field of the whole array, $d$ is the aperture diameter, which has an area $A_{R}=\pi d^{2} / 4$. The aperture collects the light from an area $A_{\text {IFOV }}=\gamma \pi r^{2} \phi_{\text {pixel }}^{2} / 4$ on the target, where $\gamma$ is the effective detector fill-factor, $A_{\text {spo }}$ is the projected illuminated area by laser and it equal to $\pi r^{2} \theta^{2} / 4$.

The mean number of photo-electrons generated from background radiation in the measurement bin time interval can be given from the following equation [4];

$$
\bar{n}_{\text {hg }}=I_{\text {stm }} \frac{\lambda}{h c} \eta K_{a t} \Psi \theta^{2}(\rho / \pi) A_{R} \beta \tau
$$

where, $I_{s t m}$ is the spectral irradiance of the sun, $K_{\text {a }}$ is the total atmospheric, and system attenuation, $\psi$ is the cosine of target orientation, and $\beta$ is the bandpass of the spectral filter.

Assuming $\rho=0.5, V=10 \mathrm{~km}$ (i.e., almost clear weather), $I_{s u n} \approx 653 \mathrm{Wm}^{-2} \mathrm{um}^{-1}$ at $1.064 \mathrm{um} \mathrm{[19],}$ $K_{a}=0.2, \psi=\cos \left(45^{\circ}\right)=0.7071$ at $45^{\circ}, \beta=1 \mathrm{~nm}$. The mean number of photo-electrons generated from background radiation in the measurement bin time interval is $\bar{n}_{h g}=0.0125 /$ bin time based on assumed system parameters. The values of the quantum efficiency or the value of target reflectivity might be inexact, but that variance will only appear as a scaling of the number of generated photo-electrons, that can be compensated by changing the transmitted laser pulse energy. APD that fabricated from silicon have weak response at $1064 \mathrm{~nm}$
[6]. Highly sensitive linear APDs manufactured from $\mathrm{HgCdTe}$, InGaAs/InP, and InGaAs/InAIAs have also been reported that may be compatible with photon counting. In particular, electron avalanche $\mathrm{HgCdTe}$ APDs should be capable of linear photon counting at $1064 \mathrm{~nm}$ [8].

Fig. I illustrates the mean number of photo-electrons generated as a function of target range bin $b$, for different values of transmitted laser energy.

In the following analysis the transmitted energy will be divide into two groups, the first one will result in received signal that will be used to examine the APD in linear mode (i.e., transmitted energy of 150, 100, $50 \mathrm{uJ}$ ), and the second one of them will result in received signal that will be used to examine the APD in photon counting modes.

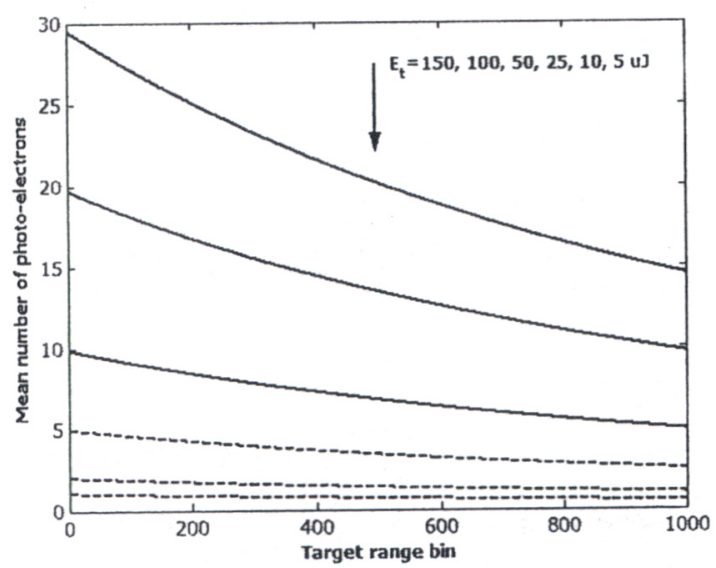

Fig. 1: Mean number of photo-electrons generated as a function of transmitted laser energy; measurement gat start from $500 \mathrm{~m}$ with range gate of $200 \mathrm{~m}$; range resolution is $20 \mathrm{~cm}$. The range to the target $r$ is related to target range bin $B$ as $r=r_{s}+B . \Delta r$. Solid curves will be used in the analysis of linear mode $A P D$, dashed curves will be used in the analysis of Geiger mode $A P D$, and linear mode $S P-A P D$. 
By using Yura's simple $r^{-2}$ approximation [10] assuming resolved target, $M$ can be computed as $M \approx 1+\frac{A_{R} A_{\| \cdot() !}}{\lambda^{2} r^{2}}$. Based assumed parameters, $M$ will equal to 154.7185 . the value of $M / \bar{N}_{s}$ is between 5.2 and 63.5 for received transmitted energy of $150 \mathrm{uJ}$ at range bin 1, and received transmitted energy of $5 \mathrm{uJ}$ at range bin 1000 respectively. Fig. 2 shows the matching between Poisson and negative-binomial distributions curves for the two cases. So under the previous two conditions the negative-binomial distribution reduces to Poisson signal statistics as follow;

$P_{S}(k)=\frac{\bar{N}_{s}^{k}}{k !} \exp \left(-\bar{N}_{S}\right)$

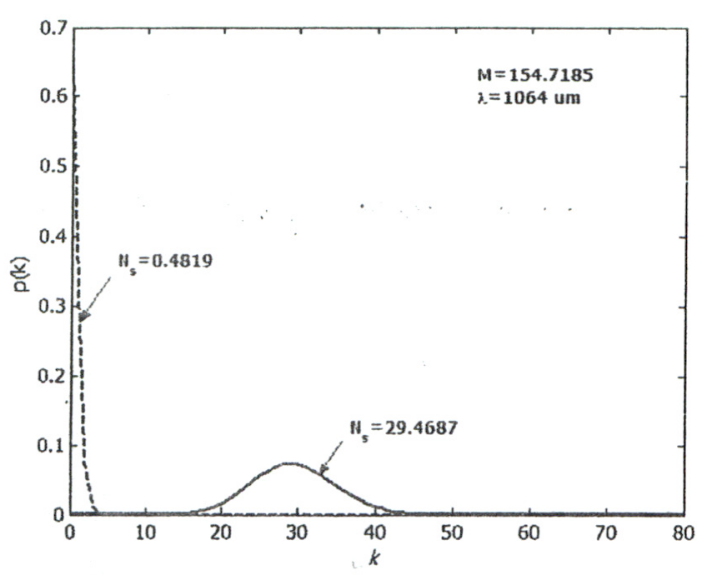

Fig. 2: negative-binomial distribution and Poisson distribution for $M / \bar{N}_{s}=5.2$ (solid curve), $M / \bar{N}_{s}=63.5$ (dashed curve).

\section{APD in Linear Mode Operation}

Use of an APD detector in linear mode causes an excess noise in the APD output due to the randomness of the APD gain. The excess noise is usually characterized by an excess noise factor, which is the ratio of the actual noise generated in an APD to the noise that would exist if all carrier pairs were multiplied by exactly $G$, where $G$ is the average APD gain. The excess noise factor is related to the APD device parameters by the following relation [11];

$F=k_{e f f} G+\left(2-\frac{1}{G}\right)\left(1-k_{c f f}\right)$ where $k_{c f f}$ is the ratio of the ionization coefficients of holes and electrons in the multiplication region of the APD. The smallest value of the excess noise factor is two for ideal APDs with $k_{\text {eff }} \rightarrow 0$ and $G \rightarrow \infty$. In practice, the output signal from an APD is still too small to be conveniently used and it must be further amplified for subsequent signal processing. Each amplifier adds thermal noise to the output but the dominate thermal noise source is from the first stage amplifier which is often called the preamplifier.

\subsection{Noise Modeling}

\subsubsection{Signal to Noise Ratio.}

The output signal to noise ratio (SNR) from APD amplifier can be written in the following form [2, 12];

$$
S N R=\frac{\left(G_{p} \bar{N}_{s}\right)^{2}}{F G^{2}\left(\bar{N}_{s}+\bar{n}_{b s}\right)+F_{c l} G_{d}^{2} \frac{I_{h} \tau}{e}+\frac{I_{s} \tau}{e}+\sigma_{c m p}^{2}}
$$

where $G_{p}$ is the photo-electron gain, $\bar{N}_{s}, \bar{n}_{h g}$ are the mean number of photo-electrons generated from the received signal and background respectively in the measurement interval $\tau, I_{s}$ and $I_{h}$ are the APD surface and bulk leakage currents respectively, the surface dark current is not multiplied because it doesn't flow in the avalanche region, but the bulk dark current is multiplied because it flow in the avalanche region. $e$ is the electron charge, and $\sigma_{\text {amp }}^{2}$, is the variance of the total amplifier noise equivalent current at the input of the preamplifier integrated over $\tau$.The total amplifier noise can then be written, to a good approximation, as $\sigma_{\text {amp }}^{2} \approx I_{\text {amp }} \tau / e$, where $I_{c m p}=2 K T / e R_{l}$ is the amplifier noise equivalent current [2]. $G_{d}, F_{d}$ is mean gain and excess noise factor respectively of dark-generated criers, their derivations are given by Hakim et al $[12,13]$, where $G_{d}=\left(G_{p}-1\right) /(\alpha w)$,

$F_{d}=\left[\left(1+\alpha w G_{d}\right)\left(1+k_{e f f} \alpha w G_{d l}\right)\right] / G_{d l}$. and $\alpha w=\ln \left[\left(1-k_{\text {eff }}\right) / G_{p}+k_{\text {eff }}\right] /\left(k_{\text {cff }}-1\right) \quad$ where $\alpha w=\ln \left[\left(1-k_{\text {cef }}\right) / G_{p}+k_{\text {etf }}\right] /\left(k_{\text {cff }}-1\right)$ is the electron ionization rate multiplied by depletion width. 


\section{E. 58 M. B. El-Mashade, H. Konber, M. Ashour and A. Abo-El-Ezz}

3.1.2 Statistical Model of Noise and Probability of False Alarm.

The noise electrons at the output of the APD amplifier are the sum of the amplified background photoelectrons, electronic noise carsed by the APD total dark current, and amplifier noise electrans.

Based on the central limit theorem, the electronic noises are assumed to be zero-mean Gaussian process with a noise variance equal to the sum of the individual noise variances as follow;

$p_{e}(m)=\frac{1}{\sqrt{2 \pi \sigma_{n}^{2}}} \exp \left(-\frac{(m)^{2}}{2 \sigma_{n}^{2}}\right)$

where $m$ represents the output noise electrons from the APD amplifier, and $\sigma_{n}^{2}$ represent the sum of all noise contributions of APD dark current, and amplifier noise electrons variances. $\sigma_{n}^{2}$ can be given as;

$$
\sigma_{n}^{2}=F_{d} G_{d}{ }^{2} \frac{I_{h} \tau}{e}+\frac{I_{s} \tau}{e}+\frac{2 K T \tau}{e^{2} R_{f}}
$$

The discrete PDF that represent the distribution of background photoelectrons is assumed to be Poisson as follow;

$$
p_{\text {bg }}(k)=\frac{\left(\bar{n}_{h g}\right)^{k}}{k !} \exp \left(-\bar{n}_{\text {bg }}\right)
$$

The probability of obtaining $m$ multiplied electrons given $k$ primary photo electrons as a function of average gain $G$, ionization coefficient ratio $k_{\text {eff }}$, was found by Mcintyre [14] as follow;

$$
p_{M c}(m \mid k)=\frac{k \cdot \Gamma\left(\frac{m}{1-k}+1\right)}{m \cdot(m-k) ! \Gamma\left(\frac{m k_{e f f}}{1-k_{e f f}}+k+1\right)}\left(\frac{1+k_{e f f}(G-1)}{G}\right)^{k+\frac{k_{e f f} m}{1-k_{c f f}}}\left(\frac{(1-k)(G-1)}{G}\right)^{m-k}
$$

then, the probability of obtaining $m$ multiplied electrons given the mean number of background photoelectrons will be given as follow;

$$
\begin{aligned}
p\left(m \mid \bar{n}_{h g}\right) & =\sum_{k=1}^{\infty} p_{M c}(m \mid k) p_{h g}(k) \\
& =\sum_{k=1}^{\infty} \frac{k \cdot \Gamma\left(\frac{m}{1-k}+1\right)}{m \cdot(m-k) ! \Gamma\left(\frac{m k_{e f f}}{1-k_{e f f}}+k+1\right)}\left(\frac{1+k_{e}(G-1)}{G}\right)^{k+\frac{m k_{e f f f}}{1-k_{e f f}}}, m>0 \\
& \times\left(\frac{(1-k)(G-1)}{G}\right)^{m-k} \times \frac{\left(\bar{n}_{h g}\right)^{k}}{k !} \exp \left(-\bar{n}_{h g}\right)
\end{aligned}
$$

The probability of $m=0$ is the same as the probability of $k=0$ given $\bar{n}_{\text {hg }}$. Total noise probability density function can be computed by convolving Eqs. (11) and (7) by using numerical convolution. Since the characteristic function of any probability density function is its Fourier transform, then by getting the discrete Fourier transform for each probability density function and getting the inverse of there multiplication, we can find the probability density function numerically as follow;

$p_{n}(m)=\operatorname{IFFT}\left(F F T\left[p\left(m \mid \bar{n}_{h g}\right)\right] \times F F T\left[p_{e}(m)\right]\right)$ 
The probability of false alarm (PFA) can be determined by summing Eq. (12) from the value of $m$ that satisfy our threshold value to infinity as follow;

$$
P_{l \cdot A}=\sum_{m=m m_{l h}}^{\infty} p_{n}(m)
$$

\subsection{Probability of signal plus noise and Target detection probability}

In the same way, the probability of multiplied photoelectrons (signal plus background) will be given by the following equation;

$$
\begin{aligned}
p_{s+b g}(m) & =\sum_{k=1}^{\infty} p_{M c}(m \mid k) p_{s+b g}(k) \\
& =\sum_{k=1}^{\infty} \frac{k \cdot \Gamma\left(\frac{m}{1-k}+1\right)}{m \cdot(m-k) ! \Gamma\left(\frac{m k_{e f f}}{1-k_{e f f}}+k+1\right)}\left(\frac{1+k_{e}(G-1)}{G}\right)^{k+\frac{m k_{e f f f}}{1-k_{\text {eff }}}} \\
& \times\left(\frac{(1-k)(G-1)}{G}\right)^{m-k} \times \frac{\left(\bar{N}_{s}+\bar{n}_{b g}\right)^{k}}{k !} \exp \left(-\left(\bar{N}_{s}+\bar{n}_{b g}\right)\right)
\end{aligned}
$$

Webb, McIntyre, and Conradi [15] proposed a simpler approximation to Eq.(14) that is applicable only in the case of Poisson distributed primary photoelectrons. This simpler probability distribution is given by;

$p(x)=\frac{1}{\sqrt{2 \pi}(1+x / \lambda)^{3 / 2}} \exp \left(\frac{-x^{2}}{2(1+x / \lambda)^{3 / 2}}\right)$

where, $x=\frac{m-G \bar{N}}{\sqrt{F G^{2} \bar{N}}}, \lambda=\frac{\sqrt{\bar{N} F}}{F-1}$, and $\bar{N}=\bar{N}_{s}+\bar{n}_{b g}$

Eq.(15) can be written in terms of multiplied electrons $m$ by using the standard method for changing statistical variables as follow;

$$
p_{W M C}(m)=\left.p(x) \frac{d x}{d m}\right|_{x \rightarrow m-\bar{N} G / \sqrt{N G^{2} F}} \quad \frac{d x}{d m}=\frac{1}{\sqrt{\bar{N} G^{2} F}}
$$

so we will have;

$$
p_{W M N^{\prime}}\left(m \mid \bar{N}_{s}, \bar{n}_{b_{R}}\right)=\frac{1}{\sqrt{2 \pi \bar{N} G^{2} F}\left[1+\frac{(m-\bar{N} G)(F-1)}{\bar{N} G F}\right]^{3 / 2}} \times \exp \left(-\frac{(m-\bar{N} G)^{2}}{2 \bar{N} G^{2} F} \frac{1}{1+\frac{(m-\bar{N} G)(F-1)}{\bar{N} G F}}\right)
$$

Fig. 3 shows the comparison between WMC approximation and McIntyre equation assuming APD with gain $\mathrm{G}=100$, and ionization coefficient ratio $k_{\text {eff }}=0.02$ [9]. From Fig. 3 we can note that WMC is very good approximation to the McIntyre distribution especially for values of $\bar{N}$ greater than 3 , then the probability density function of signal plus noise (background noise and electronic noise) can be given by the following equation; 


\section{E. 60 M. B. El-Mashade, H. Konber, M. Ashour and A. Abo-El-Ezz}

$p_{s+n}(m)=\operatorname{IFFT}\left(\operatorname{FFT}\left[p_{W M C^{\cdot}}\left(m \mid \bar{N}_{s}+\bar{n}_{b g}\right)\right] \times \operatorname{FFT}\left[p_{n}(m)\right]\right)$

where $p_{n}(m)$ is given by Eq. (12).

The probability of detection can be calculated as follow;

$P_{D}=\sum_{m=m_{t h}}^{\infty} p_{s+n}(m)$

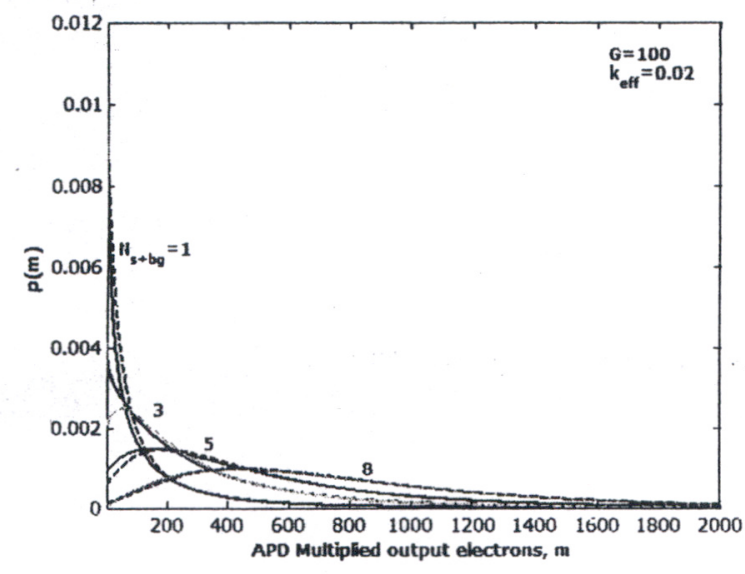

Fig. 3: Probability of signal plus background noise as a function of APD multiplied output electrons (m) by using WMC approximation (dashed curves) and McIntyre density function (solid curves).

\subsection{Numerical Simulation results for linear mode APD}

By using the assumed APD data in Table I [3], we can find that $\sigma_{n}^{2}=1897.28$.

\begin{tabular}{|l|c|}
\hline \multicolumn{2}{|l|}{ Table I. Assumed parameter of APD } \\
\hline Ionization ratio & 0.02 \\
\hline photoelectron gain & 100 \\
\hline surface leakage current & $100 \mathrm{nA}$ \\
\hline bulk leakage current & $10 \mathrm{pA}$ \\
\hline$F_{p}$ & 3.9502 \\
\hline$F_{d}$ & 10.11 \\
\hline$G_{d}$ & 29.4774 \\
\hline $\begin{array}{l}\text { Amplifier noise equivalent } \\
\text { current }\end{array}$ & $100 \mathrm{nA}$ \\
\hline
\end{tabular}

Fig. 4 shows the probability of signal plus noise $p_{s+n}(m)$ by using Eq. (17). We can note from that figure, as the mean number of electrons at the output of the amplifier increase, the probability of signal plus noise can be approximated by Gaussian distribution.

Fig. 5 shows the amplifier output noise electrons probability density function by using numerical convolution (i.e., Eq. (12)). Fig. 6 shows the result of PFA as a function of threshold electrons $m$. From Fig. 6, the threshold under numerical convolution is 922 electrons for PFA of $10^{-4}$.

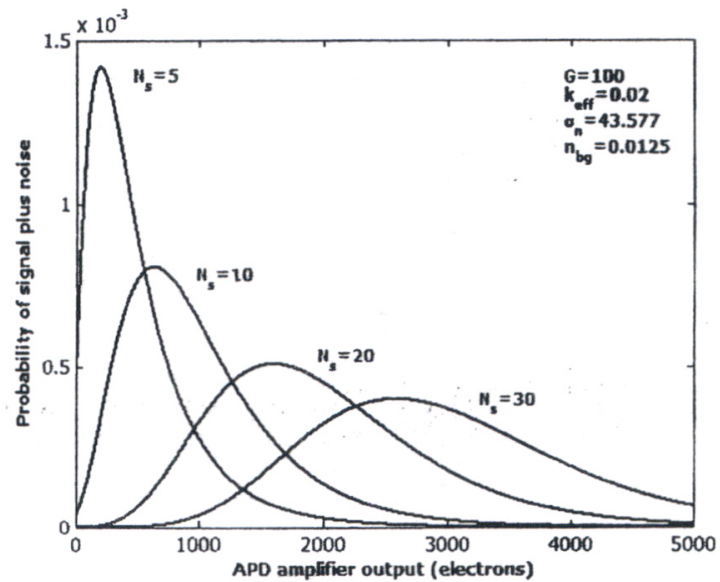

Fig. 4: Probability of signal plus noise at APD amplifier output.

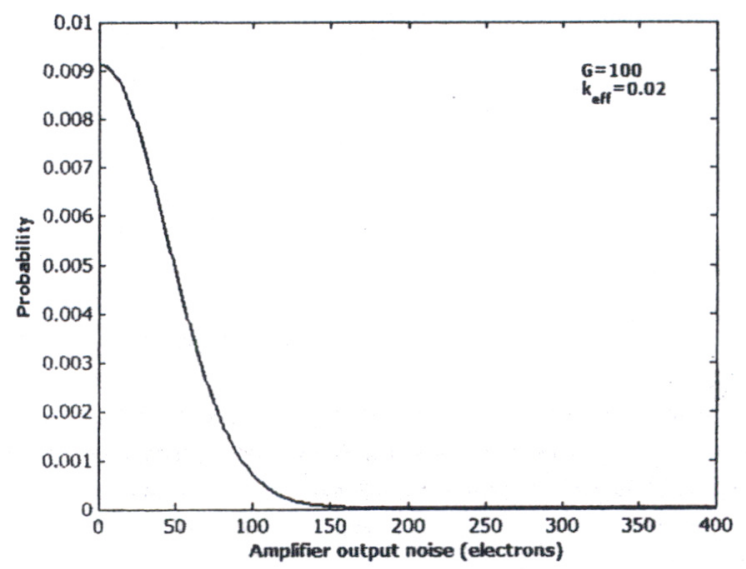

Fig. 5: Noise probability density function by using numerical convolution.

Fig. 7 shows the probability of detection as a function in target range bin number with threshold of 922 electrons. From Fig. 7 we can note that the analog mode of operation has good performance for mean photo-electrons greater 
than ten photo-electrons (solid curves in Fig. 1), and have bad performance for small values of mean photo-electrons (i.e., low count rate operation). So this mode of operation is suitable only for high count rate operation. Low count rate operation require more sensitivity for one photoelectron, such as Geiger-mode APD operation, or Linear mode single photon APD operation. It is very important to note that in our analysis, the APD gain is not optimized for maximum SNR.

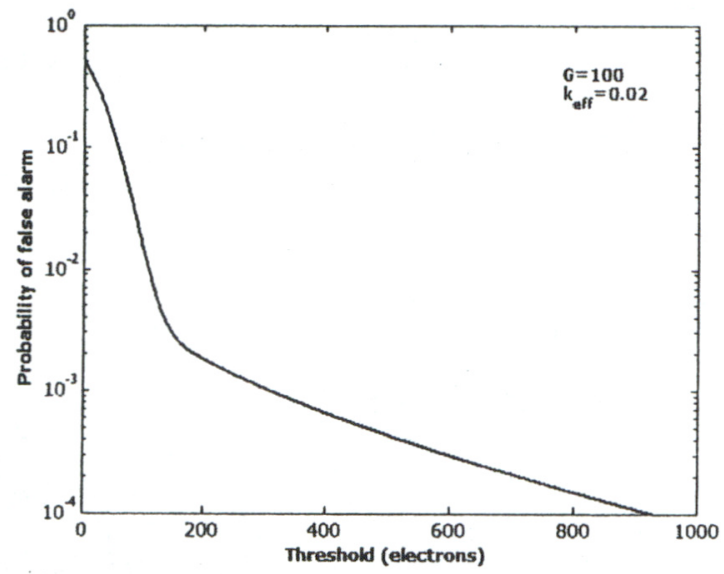

Fig. 6: Probability of false alarm versus threshold setting.

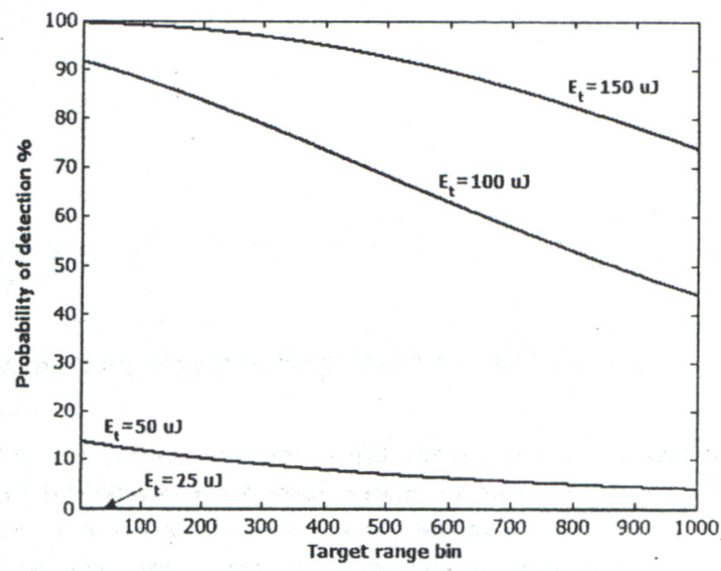

Fig. 7: Target detection probability versus target range bin number, threshold is 922 electrons. Measurement gat start from $500 \mathrm{~m}$ with range gate of $200 \mathrm{~m}$; range resolution is 20 $\mathrm{cm}$. The range to the target $r$ is related to target range bin $B$ as $r=r_{s}+B . \Delta r$.

\section{APD in Geiger-mode operation}

In Geiger mode, the reverse bias across an APD is so high that the rate of creation of charge carriers by impact ionization exceeds the rate at which the charge carriers can be extracted from the device, and the output current quickly surges to a saturated value set by circuit parameters. A single electron can initiate the current surge, or firing. The term primary electron will be used for an electron that initiates a firing. Three Poisson processes create primary electrons, which are created by absorption of laser photons reflected from the target, absorption of background photons (i.e., reflected sunlight), and dark current electrons, which are created by thermal effects within the detector material. These processes are statistically independent of one another as long as the rate of primary-electron creation is not saturated.

\subsection{Detection Model by Typical Geiger-Mode APD}

The APD is biased into Geiger mode for a particular time interval, (measurement gate time), on every laser pulse which is divided into discrete bins, each with the same time duration. Above the breakdown voltage, Geiger-Mode APD is characterized by the breakdown probability, which is the probability that a single primary carrier into the multiplication layer of the APD will trigger the avalanche breakdown. The ideal case and also the worst case, is that the detector fires in response to the first primary electron created within the gate (i.e., breakdown probability equal to one), so our threshold value is one electron $\left(k_{t h}=1\right)$. Thus, if the detector fires from a noise source before laser photons reflected from the target arrive, then the detector will not respond to the target return for that laser pulse.

\subsubsection{Probability of detection for Geiger mode operation (single pulse).}

Define $\bar{N}_{s}$ as the mean number of target return photoelectrons created within the bin duration time. Assume that the mean rates of primary electrons creation by absorption of background photons and by dark current are constant during data collection. The combined mean rate of noise can be defined as $\bar{N}_{n}=r b \tau$, with units of primary electrons per gate interval, where $r$ is the noise rate, $b$ is the number of range bins, and $\tau$ is the bin duration time, then the noise contribution to all range bins $\bar{n}_{n}=\bar{N}_{n} / b$

Because the detector fires in response to the first primary electron (from signal or noise), the only way for 


\section{E. 62 M. B. El-Mashade, H. Konber, M. Ashour and A. Abo-El-Ezz}

firing to occur in the bin $B$ is to have no primary electrons in the first $B-1$ bing $(k=0)$, and at least one primary electron $\left(k_{t h}=1\right)$ in the bin $B$.

Therefore the probability of detecting signal in presence of noise in range bin $B$ is given by [4];

$P_{D}(B)=\left[\prod_{B=1}^{B-1} P(k=0)\right] P(k \geq 1)$

The first term is the probability that no noise electrons at any bin created before returned target signal bin, and it

$$
\begin{aligned}
P_{D}(B) & =\left[\prod_{B=1}^{B-1} \exp \left(-\bar{n}_{n}\right)\right] \sum_{k=1}^{\infty} \frac{\left(\bar{N}_{s}+\bar{n}_{n}\right)^{k} \exp \left(-\left(N_{s}+\bar{n}_{n}\right)\right)}{k !} \\
& =\exp \left[-(B-1) \bar{n}_{n}\right]\left[1-\exp \left(-\bar{N}_{s}-\bar{n}_{n}\right)\right]
\end{aligned}
$$

Eq. (20) shows that the probability of firing in the target bin is reduced by the factor $\exp \left[-(B-1) \bar{n}_{n}\right]$, where $(B-1) \bar{n}_{n}$ is the mean number of noise primary electrons created within the gate before the arrival of laser photons from the target. So, to reduce the noise affect before returned target signal, the value of $(B-1) \bar{n}_{n}$ must be much less than one primary electron.

\subsubsection{Probability of false alarm (single pulse).}

Define the single pulse PFA as the probability that the detector fires in the one of the non-target bins in the present of target [4]. Then PFA is $1-P_{D}(B)-\exp \left(-\bar{N}_{s}-\bar{n}_{n}\right)$, where the last term is the probability that the detector does not fire from the target return nor from noise and it is called the probability of pixel drop-out [5].

\subsection{Multiple-pulse detection and false alarm probabilities}

On of the most method to enhance the system performance is to process multiple pulses that increase the detection probabilities and decrease the false alarm probabilities. Binary integration algorithm will be used in the following analysis. In this algorithm thresholding and detection are done pulse by pulse followed by a detection criteria based on the number of detected pulses out of transmitted pulses $U$.

The criteria in [5] will be used; Let $x$, be the probability that the GM-APD fires in the range bin number $\mathrm{j}$ so we will have the following three cases; holds because the number of events in any bin is independent of the number in any other bin. Second term is the probability that target photo-electrons signal plus noise electrons exceeds one primary electron, so we will have following form;

$$
\begin{aligned}
& x_{j}=\exp \left[-(j-1) \bar{n}_{n}\right]\left[1-\exp \left(-\bar{n}_{n}\right)\right], \quad j<B, \\
& x_{j}=\exp \left[-(B-1) \bar{n}_{n}\right]\left[1-\exp \left(\bar{N}_{s}-\bar{n}_{n}\right)\right], j=B, \\
& x_{j}=\exp \left[-(B-1) \bar{n}_{n}-\bar{N}_{s}\right]\left[1-\exp \left(-\bar{n}_{n}\right)\right], j>B
\end{aligned}
$$

The probability of detection is the probability that the number of firings in the target bin is $\geq T$, where $T$ is threshold of the number of detected pulses, so the probability of detection will be given by;

$$
P_{D}=1-\sum_{i=0}^{T-1} \frac{U !}{(U-i) ! i !} x_{B}^{\prime}\left(1-x_{B}\right)^{1-1}
$$

The probability of false alarm in multiple pulse processing case is defined as the probability that one or more of nontarget bin has $\geq T$ firings and will be given by;

$$
\begin{aligned}
P_{l: 4}= & \sum_{j=1}^{B-1}\left[1-\sum_{i=0}^{T-1} \frac{U !}{(U-i) i !}\left(1-x_{l}\right)^{(i-i} x_{j}^{\prime}\right] \\
& +\sum_{j=B+1}^{h}\left[1-\sum_{i=0}^{T-1} \frac{U !}{(U-i) i !}\left(1-x_{l}\right)^{(I-1} x_{j}^{\prime}\right]
\end{aligned}
$$




\section{Linear Mode Single Photon APD}

In contrast to GM-APD, APDs may be used to count single photons while being biased below the breakdown point as in linear mode. The photocurrent pulses resulting from a single photon absorption can be detected via a discriminator threshold crossing with a relatively high probability as long as the APD gain is sufficiently large and the circuit thermal noise is relatively low. There is no after pulsing since the APD is operating in its linear regime. The APD bias voltage does not need to be restored after each photo-electron detection.

\subsection{Detection Model by Typical Linear-Mode Single Photon APD}

The model used for this mode of operation is shown in Fig. 8. The APD is exactly the same as that in the linear mode except that the average APD gain is set to the highest achievable value, and the preamplifier used is an ultra low noise amplifier, this requires some form of APD temperature control and a very stable bias voltage supply. The output of the preamplifier is compared against a threshold with the use of a comparator whose output is connected to an electrical pulse counter. The threshold level of the comparator has to be carefully set in order to achieve a high single photoelectron detection efficiency while maintaining a tolerable noise count rate. The signal photo-électrons and the number of dark noise electrons are both assumed to be Poisson processes.

\begin{tabular}{|c|c|c|}
\hline $\begin{array}{l}\text { APD } \\
V_{t x}<V_{\text {Brat - Sewn }}\end{array}$ & $\begin{array}{l}\text { Pre-Amplifier } \\
\& \text { other } \\
\text { electronics }\end{array}$ & $\begin{array}{l}\text { Threshold } \\
\text { \& Decision }\end{array}$ \\
\hline
\end{tabular}

Fig. 8: $L A D A R$ direct detection receiver model based on linear mode APD photon counter.

\subsubsection{Single photo-electron detection probability.}

The probability that single primary carrier will be detected by linear mode APD is the probability that a given primary photocarrier $(k=1)$ result in an amplified signal greater than the receiver's threshold $m_{t h}$;

$P_{\text {sin } g l e}=1-\sum_{m=k}^{m_{l h}} p_{M}(m \mid k), k=1$

where the probability of multiplied electrons $m$ given one primary photoelectron can be found from McIntyre distribution (i.e.. Eq. (10)) by letting $k=1$.
The probability that an APD can detect a single photoelectron is plotted in Fig. 9 for fixed value of $k_{\text {eff }}$ and various values of gain.

We can note from Fig, 9 that low effective ionization value and high gain can improve the single photo electron detection efficiency. Gain with the value 8000 , and $k_{\text {eff }}$ equal 0.0064 has been achieved experimentally [18]. APD with $G=8000, k_{\text {eff }}=0.0064$ will be assumed in the following analysis.

\subsection{Noise Model and threshold setting}

There are two source of noise in that system; Dark current noise and circuit thermal noise; The mean number of primary noise electrons generated per bin time $\tau$ just depend on the average noise rate $r$ caused by dark current and incoherent background radiation according to $\bar{n}_{n}=q \times r$ with units of primary electrons per measurement interval (bin interval). The number of primary dark electron counts in an integration time is assumed to obey the Poisson distribution as follow;

$p_{n}\left(k \mid \bar{n}_{n}\right)=\frac{\bar{n}_{n}{ }^{k} \exp \left(-\bar{n}_{n}\right)}{k !}$

For comparison reasons with GM-APD, all primary noise electrons caused by background radiation or by dark current electrons are assumed to be multiplied with the same mean gain and same excess noise factor and the multiplied output electrons follows Mclntyre distribution. The amplifier noise current can be modeled as a zero mean Gaussian random process;

$p_{e}(k)=\frac{1}{\sqrt{2 \pi \sigma_{\text {amp }}^{2}}} \exp \left(-\frac{k^{2}}{2 \sigma_{\text {amp }}^{2}}\right), k \geq 0$

In this type of receivers (i.e., linear mode single photon APD ) the receiver threshold electrons is set to be a few times of RMS value of the amplifier noise electrons as $m_{t h}=\mathbf{f} \times \sigma_{\text {camp }}$. An ultra low noise amplifier which have about $\sigma_{\text {amp }}=20$ electrons RMS can reasonably be achieved if low-capacitance bump-bonding is used to hybridized the detector pixel to its readout circuit [6]. The probability of false alarm against amplifier noise is given by;

$P F A=\int_{k_{t h}}^{\infty} p_{n}(k) d k=\frac{1}{2} \operatorname{erfc}\left(\frac{\left(k_{t h} / \sigma_{a m n}\right)}{\sqrt{2}}\right)$ 


\section{E. 64 M. B. El-Mashade, H. Konber, M. Ashour and A. Abo-El-Ezz}

As shown in Fig. 10, threshold of 100 electron will give value of PFA against amplifier noise less than $10^{-6}$, single photo-electron detection probability of $\underline{0.687}$ at gain with the value 8000 , and $k_{\text {eff }}=0.0064$, and a factor of five margin for an ultra low noise amplifier with about 20 electrons RMS in band noise.

The probability of false alarm in certain range bin is the probability that primary noise electrons generated per bin time $\tau$ result in an amplified signal greater than the receiver's threshold $m_{t h}$ and can be given by summing the product of Eq. (25) and the probability that a given primary photocarriers result in an amplified signal greater than the receiver's threshold $m_{t h}$ over $k$ as follow;

$$
P_{F A}=\sum_{k}\left(p_{n}\left(k \mid \bar{n}_{n}\right) \times\left[1-\sum_{m=k}^{m_{t h}} p_{M c}(m \mid k)\right]\right)
$$

,$k \geq 1$
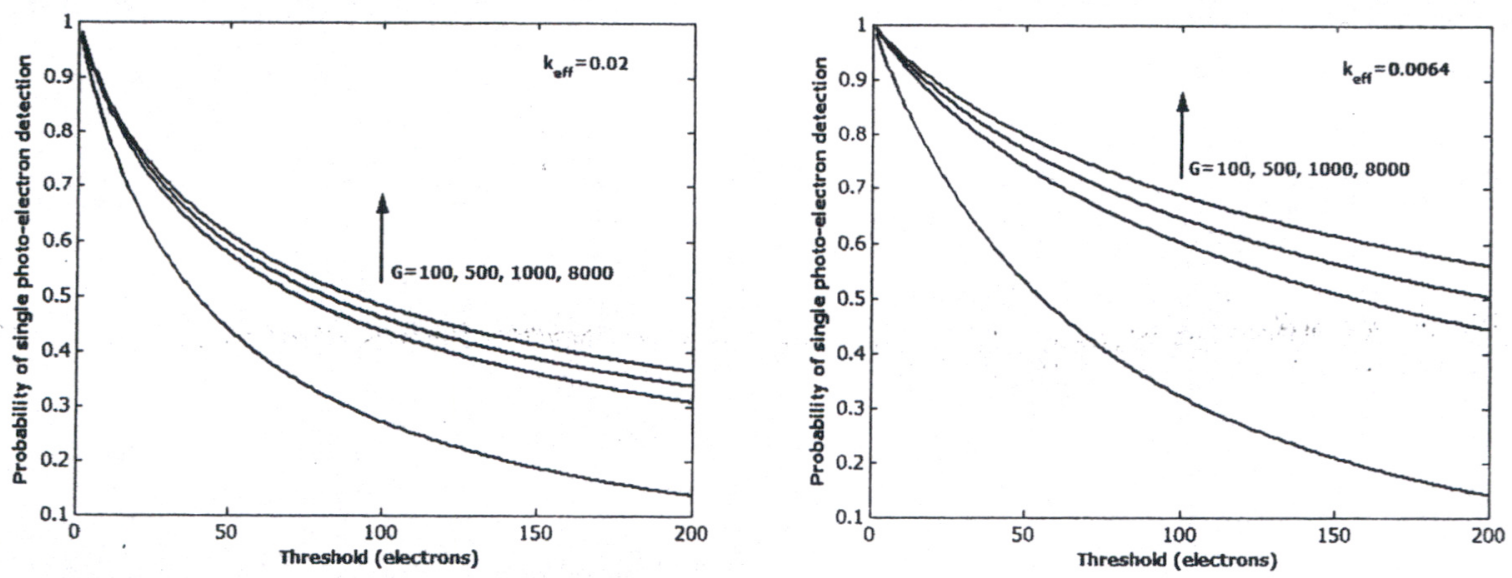

Fig. 9: single photo-electron detection probability. for effective ionization coefficient $k_{\text {cff }}=0.02$ (left), $k_{\text {eff }}=0.0064$ (right).

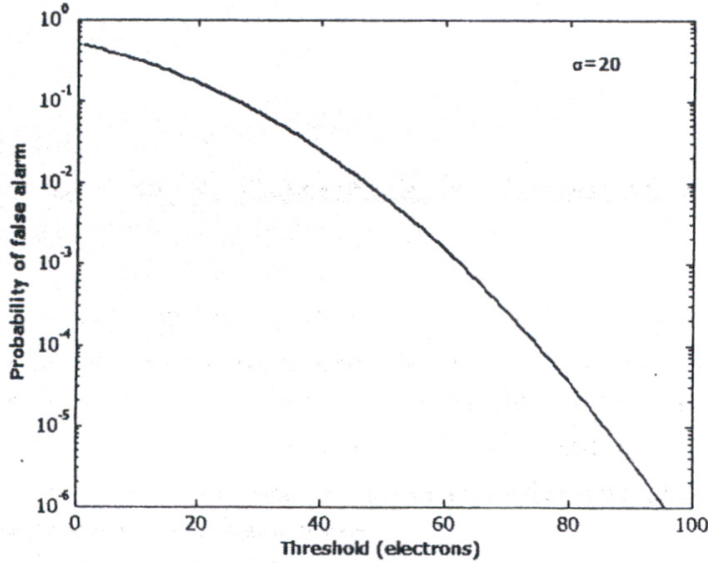

Fig. 10: Probability of false alarm against amplifier noise electrons as a function of threshold electrons.
Last expression is equivalent to the following expression;

$$
P_{F A}=1-\sum_{m=0}^{m_{l h}} p\left(m \mid \bar{n}_{n}\right)
$$

where $p\left(m=0 \mid \bar{n}_{n}\right)=p_{n}\left(k=0 \mid \bar{n}_{n}\right)$, and $p\left(m \mid \bar{n}_{n}\right)$ is the probability of obtaining $m$ multiplied noise electrons given the mean number of primary noise electrons generated per bin time $\tau$ which is given by;

$p\left(m \mid \bar{n}_{n}\right)=\sum_{k=1}^{\infty} p_{\Lambda}(m \mid k) p_{n}\left(k \mid \bar{n}_{n}\right)$

Fig. 1 I shows the probability of multiplied output noise electrons from the APD given by Eq. (30) for $G=8000$, and $k_{e f f}=0.0064$, assuming the same noise rates used in 
Geiger-mode analysis, $r_{1}=10^{4} \mathrm{~Hz}, r_{2}=10^{5} \mathrm{~Hz}$, and $r_{3}=10^{6} \mathrm{~Hz}$, and integration time of $1.33 \mathrm{~ns}$.

The total rate of false alarms in the measurement gate at the present of the target is given by;

$P_{F A}=(b-1)\left(\sum_{k}\left(\begin{array}{l}p_{n}\left(k \mid \bar{n}_{n}\right) \\ \left.\times\left[1-\sum_{m=k}^{m_{\text {th }}} p_{M}(m \mid k)\right]\right)\end{array}\right)\right)$

where $b$ is the number of target range bins. With $m_{\text {th }}=100, \mathrm{G}=8000, k_{\text {cff }}=0.0064$, the total rate of false alarms generated in the measurement gate is 0.00913 for mean noise rate of $10^{4}$, and 0.0913 for mean noise rate of $10^{5}$, and 0.9123 for mean noise rate of $10^{6}$.

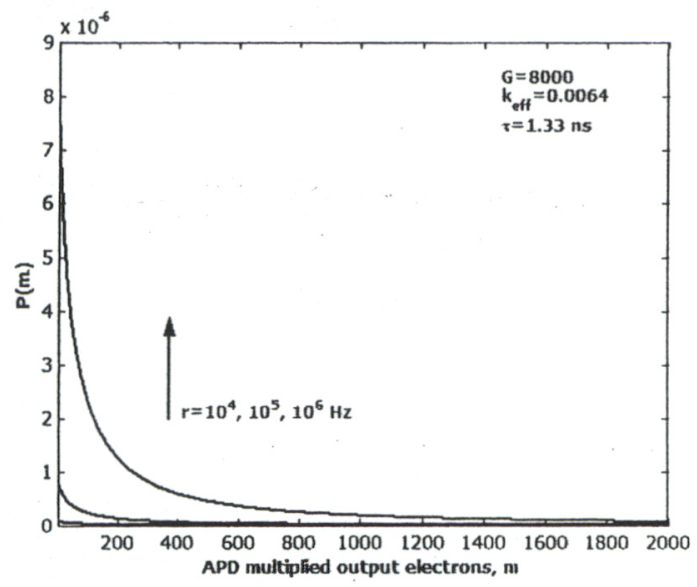

Fig. 11: The probability of multiplied output noise electrons from the APD (m) based McIntyre distribution from $m=1$ for several values of mean noise rate with mean gain 8000 , and ionization coefficient of 0.0064 .

\subsection{Target detection probability in the presence of} noise

Not as Geiger mode which affected by the generation of primary dark electrons, LM-SPAPD detection probability of signal in presence of noise in range bin $B$ depends only on the number of primary photo electrons generated in the target range bin and the threshold of the receiver as follow;

$$
P_{D}=\sum_{k}\left(\begin{array}{c}
p_{s+n}\left(k \mid \bar{n}_{n}+\bar{N}_{s}\right) \\
\times\left[1-\sum_{m=k}^{m_{t h}} p_{M}(m \mid k)\right]
\end{array}\right)
$$

or,

$P_{D}=1-\sum_{m=0}^{m_{\text {th }}} p\left(m \mid \bar{n}_{n}+\bar{N}_{s}\right)$

\subsection{Multiple-pulse detection and false alarm probabilities}

For the case of LM-SPAPD the probability of detection at certain range bin $B$ is given by;

$$
x_{j}=\sum_{k}\left(\begin{array}{c}
p_{s+n}\left(k \mid \bar{n}_{n}+\bar{N}_{s}\right) \\
\times\left[1-\sum_{m=k}^{m_{t h}} p_{M}(m \mid k)\right]
\end{array}\right), j=B
$$

and the probability of false alarm in non target bin is given by;

$$
\begin{gathered}
x_{j}=\sum_{k}\left(p_{n}\left(k \mid \bar{n}_{n}\right) \times\left[1-\sum_{m=k}^{m_{m}} p_{M}(m \mid k)\right]\right) \\
, j \neq B
\end{gathered}
$$

so the probability of obtaining at least $T$ pulses detected from $U$ transmitted pulses is given by;

$$
P_{D}=1-\sum_{i=0}^{T-1} \frac{U !}{(U-i) ! i !} x_{B}^{i}\left(1-x_{B}\right)^{\prime \prime-1}
$$

The total rate of false alarms (the probability that one or more of nontarget bin has $\geq T$ firings) will be given by;

$$
\begin{aligned}
& P_{l \cdot A}=(b-1)\left[1-\sum_{i=0}^{T-1} \frac{U !}{(U-i) i !}\left(1-x_{,}\right)^{\prime \prime-i} x_{j}^{i}\right] \\
& P_{l i d} \approx(b-1)\left[\frac{U !}{(U-i) i !} x_{i}^{r}\right], \text { where } x_{,}<1
\end{aligned}
$$




\section{E. 66 M. B. El-Mashade, H. Konber, M. Ashour and A. Abo-El-Ezz}

\section{Performance comparison}

In this section, the performance comparisons between GM-APD and LM-SPAPD is presented in terms of the probability of detection and probability of false alarm as a function of target range, transmitted laser pulse energy and mean noise rates. As in [5], three mean noise rates

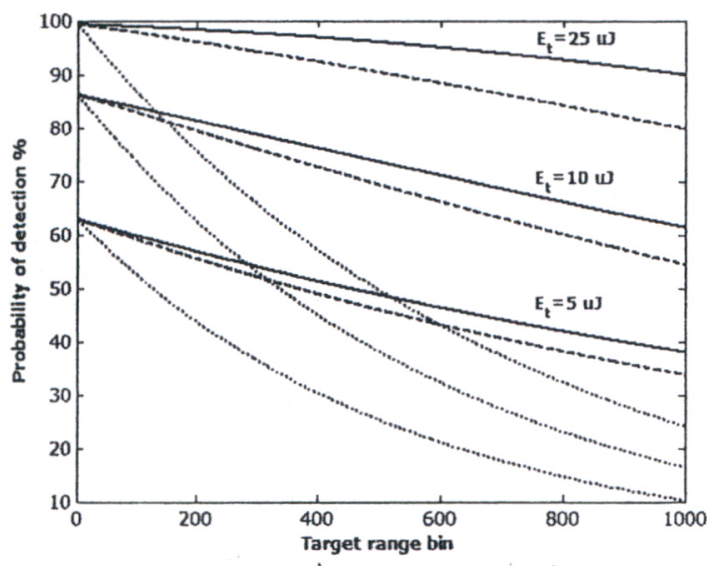

will be assumed to show the effect of reducing the mean primary noise electrons on the performance of the receiver.

Fig. 12 shows target detection probabilities for three transmitted laser pulse energies (second group of transmitted energy shown in Fig. 1) and various noise rates.

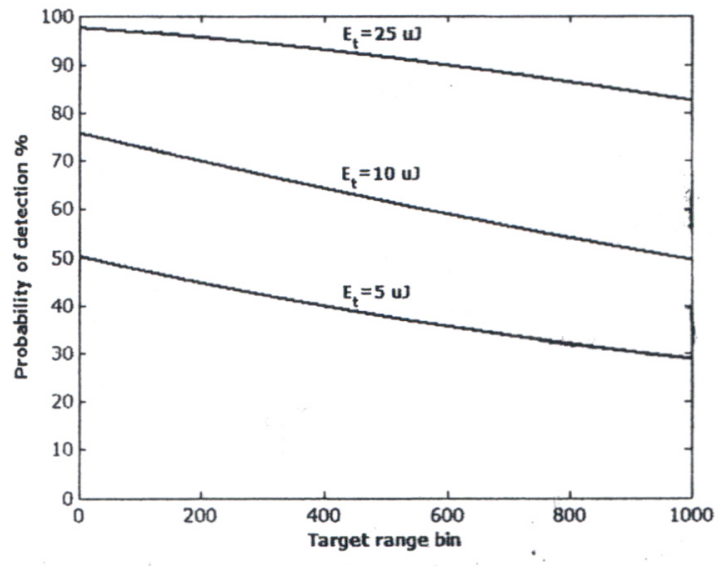

Fig. 12: Target detection probability versus target range bin number as a function of transmitted laser pulses and various mean noise rates. Measurement gat start from $500 \mathrm{~m}$ with range gate of $200 \mathrm{~m}$; range resolution is $20 \mathrm{~cm}$. GM-APD (left), LM-SPAPD assuming receiver threshold of 100 electrons, $G=8000, k_{\text {eff }}=0.0064$ (right). Solid curves are for $r=10^{4} \mathrm{~Hz}$,dashed curves are for $r=10^{5} \mathrm{~Hz}$, and dotted curves are for $r=10^{6} \mathrm{~Hz}$. Each set of curves belong to the same transmitted laser pulse energy are equal at bin number one.

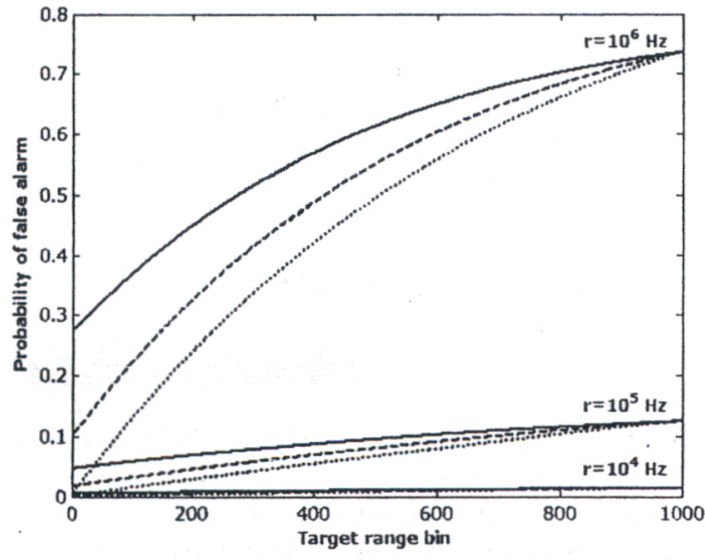

Fig. 13: Probability of false alarm versus target range bin number. Measurement gat start from $500 \mathrm{~m}$ with range gate of $200 \mathrm{~m}$; range resolution is $20 \mathrm{~cm}$. Solid curves are for $E_{1}=25 \mathrm{uJ}$, dashed curves for $E_{t}=10 \mathrm{uJ}$, and dotted curves are for $E_{1}=5 \mathrm{uJ}$.
By comparing Fig. 12a, and 12b we can note the bad effect of mean primary noise rates on the performance of the detector in Geiger-mode operation, on other hand it nearly have no effect on the target detection probability of LM-SPAPD. That is because the detector in the linearmode have no memory with earlier events (i.e., generation of primary carrier) caused by signal or by noise, but in Geiger-mode earlier events can make the detector die and must be reset to respond to another event.

Fig. 13 shows the PFA for GM-APD as a function of transmitted energy and various noise rates. PFA of LMSPAPD a function of the receiver threshold electrons is shown in Fig. 14. From Fig. 14. we can note that at threshold of 100 electrons, the probability that noise can register false alarm in certain range bin is $9.1367 \times 10^{-6}$ for mean noise rate of $10^{4}$, and $9.1364 \times 10^{-5}$ for mean noise rate of $10^{5}$, and $9.1329 \times 10^{-4}$ for mean noise rate of $10^{6}$. Figs. $12 \mathrm{a}$, and 13 show the effect of primary noise electrons on the performance of the Geiger mode receiver. 
Higher mean noise rate reduce target detection probability and increase the PFA.

Noise rates can be reduced by means as cooling the detector to reduce the dark current and using a narrow bandpass filter to reduce the background light (see Eq. (2)).

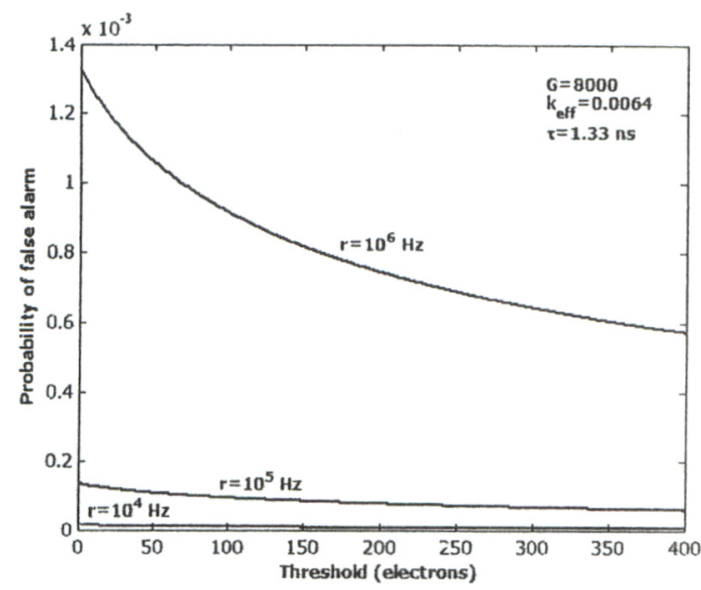

Fig. 14: Probability of false alarm as a function of threshold electrons.

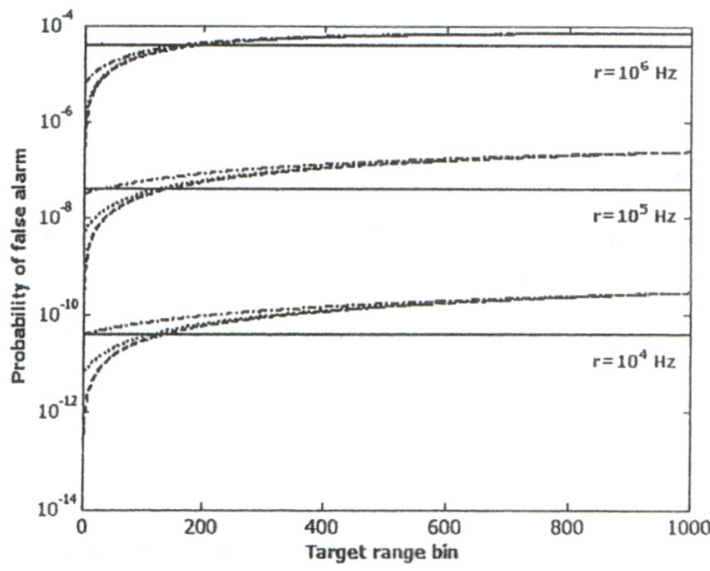

Fig. 15: Probability of false alarm as a function of target range bin number. Measurement gat start from $500 \mathrm{~m}$ with range gate of $200 \mathrm{~m}$; range resolution is $20 \mathrm{~cm}$. Number of transmitted pulses is $U=10$, and the minimum requirement of detected pulses is $T=3$. Solid curves are for LM-SPAPD for all transmitted energy and with $m_{t h}=100, G=8000, k_{\text {eff }}=0.0064$. Remaining curves are for GM-APD, where dash curves for $E_{t}=25 \mathrm{uJ}$, dot curves for $E_{t}=10 \mathrm{uJ}$, and dash-dot curves for $E_{1}=5 \mathrm{uJ}$.
Fig. 15 shows the PFA for both LM-SPAPD, and GMAPD, where the number of transmitted pulses is $U=10$, and the minimum requirement of detected pulses is $T=3$. We can note that the total rate of false alarms generated in the measurement gate in the presence of target is $3.8865 \times 10^{-11}$ for mean noise rate of $10^{4}$, and $3.8862 \times 10^{-8}$ for mean noise rate of $10^{5}$, and $3.8829 \times 10^{-5}$ for mean noise rate of $10^{6}$. This values are lower than that for GM-APD in most measurement gate and constant over measurement interval.

In Fig. 15 the curves that belong GM-APD is based on Eq. (23), but the dependence of that equation on target position $B$ and signal strength makes the calculation of PFA hard to be implemented with higher combination values of transmitted pulses and threshold (combination of $\mathrm{U}=10, \mathrm{~T}=5$ give unexpected results for GM-APD). But as $x,<x$, for all $j$ except $j=B$ and

$x_{1}=\left[1-\exp \left(-\bar{n}_{n}\right)\right]$, Eq.(23) can be simplified to give an upper limit that does not depend on the target as follow [5];

$$
P_{F A, \max }<(b-1)\left\{1-\sum_{i=0}^{r-1}\left(\begin{array}{l}
\frac{U !}{(U-i) ! i !} \times\left[\exp \left(-\bar{n}_{n}\right)\right]^{(i-i} \\
\times\left[1-\exp \left(-\bar{n}_{n}\right)\right]^{\prime}
\end{array}\right)\right\}
$$

Fig. 16. shows the probability of detection for GMAPD (left), and LM-SPAPD (right) at fixed threshold T=5 , and transmitted laser pulses $U=10$ pulses. We can note from Fig. 16 that at noise rate of $r=10^{4} \mathrm{~Hz}$, GM-APD give performance better than that for LM-SPAPD. For higher noise rates (i.e., $r=10^{5} \mathrm{~Hz}$, and $r=10^{6} \mathrm{~Hz}$ ) the probability of detection for the GM-APD is better than that belong the LM-SPAPD only at the front bins of measurement gate, but as the number of range bins increase the value of the probability of detection decrease rapidly and LM-SPAPD give performance better than that for GM-APD.

If the Schwartz roll $[16,17]$ used; that optimum threshold $\mathrm{T}$ follows approximately a relationship given by $z \sqrt{U}$, where $z$ is a constant approximately equal to 1.5 , we will have the results shown in Fig. 17 for both GMAPD (left), and LM-SPAPD (right) at $E_{,}=5 \mathrm{uJ}$, and mean noise rate $r=10^{6} \mathrm{~Hz}$.

We can note from Fig. 17 that the performance of GMAPD operation is better than that for LM-SPAPD operation only at the front range bins of the measurement 


\section{E. 68 M. B. El-Mashade, H. Konber, M. Ashour and A. Abo-El-Fzz}

gate, but as the number of range bins increase, LM-

SPAPD give performance better than that for GM-APD.
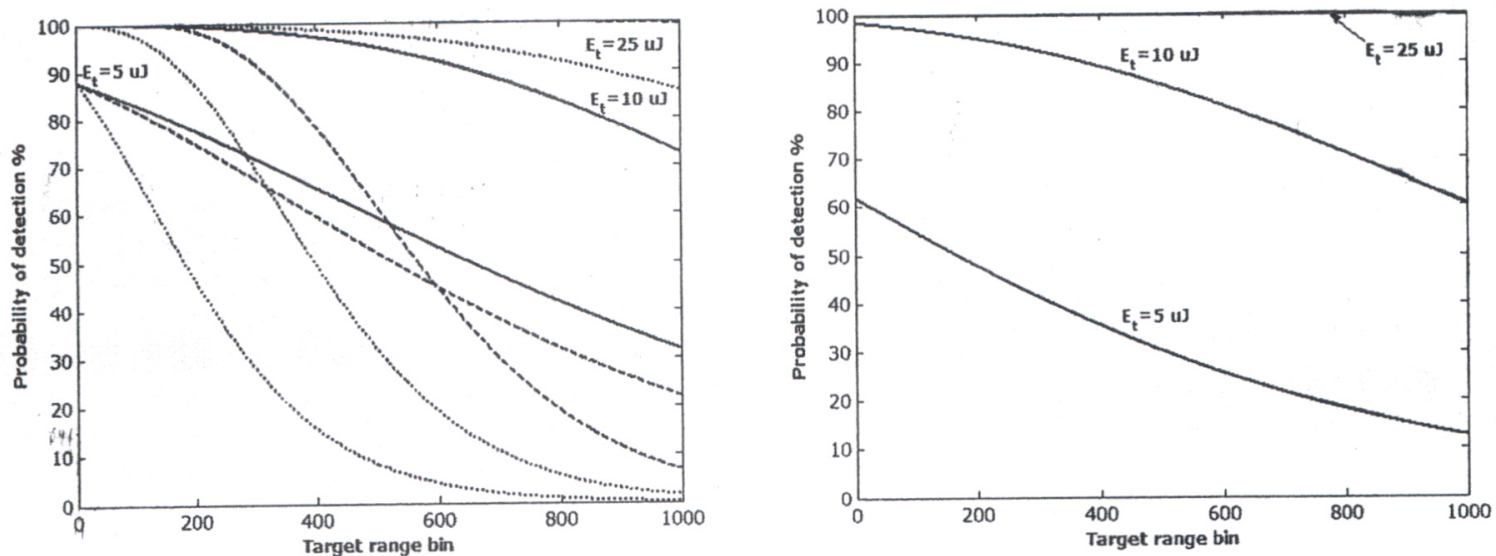

Fig. 16: Target detection probability versus target range bin number for GM-APD (left), and LM-SPAPD (right). (U=10, T=5), measurement gat start from $500 \mathrm{~m}$ with range gate of $200 \mathrm{~m}$; range resolution is $20 \mathrm{~cm}$. Solid curves are for $r=10^{4} \mathrm{~Hz}$, dashed curves are for $r=10^{5} \mathrm{~Hz}$, and dotted curves are for $r=10^{6} \mathrm{~Hz}$. Curves group of $25 \mathrm{uJ}$ transmitted energy are not shown except for $r=10^{4} \mathrm{~Hz}$.
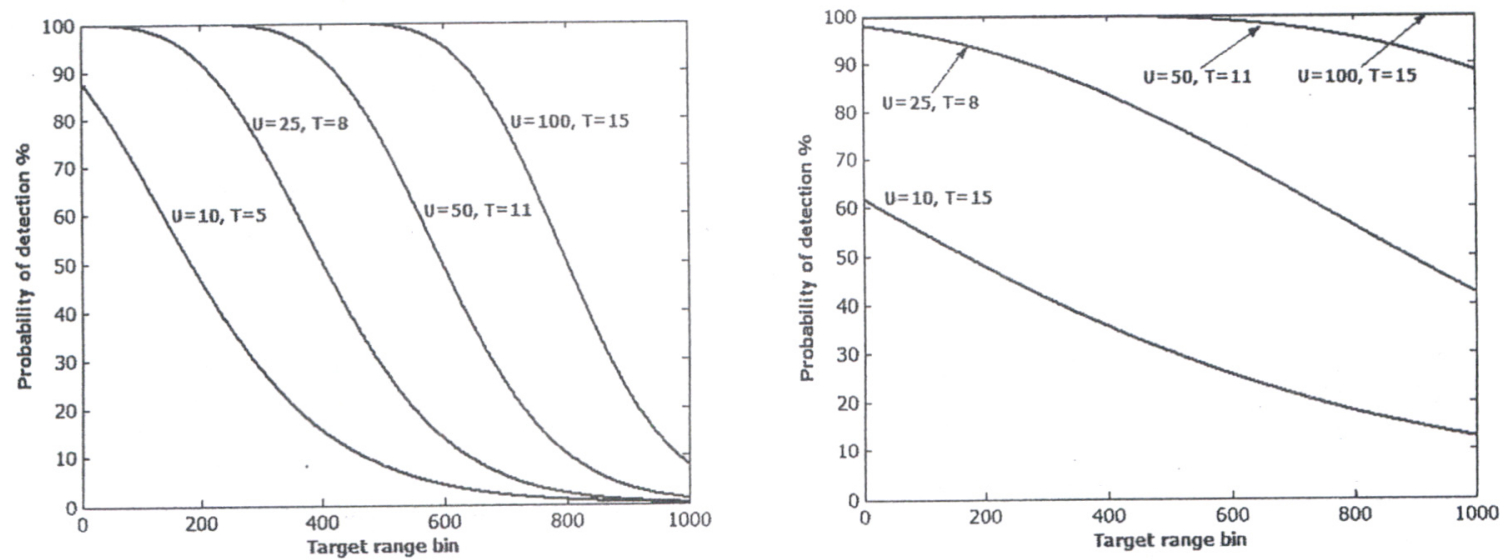

Fig. 17: Target detection probability versus target range bin number for GM-APD (left), and LM-SPAPD (right) at $E_{1}=5 u J$, and mean noise rate $r=10^{6} \mathrm{~Hz}$. Measurement gat start from $500 \mathrm{~m}$ with range gate of $200 \mathrm{~m}$; range resolution is $20 \mathrm{~cm}$. Optimum threshold $T$ follows Schwartz roll for each transmitted pulses $U$ (upper). Fixed threshed and different transmitted pulses (lower). measurement gat start from $500 \mathrm{~m}$ with range gate of $200 \mathrm{~m}$; range resolution is $20 \mathrm{~cm}$.

\section{Conclusions}

In this paper, we provide a complete detection performance of the Ladar system that uses an Avalanche diode as the basic element for the signal detection under different operating conditions. This laser system has many applications in the defense field concerning target detection and identification. The extraction of these features depends on the processing algorithms, target properties and $3 \mathrm{D}$ images quality. It was shown that 
Avalanche photodiode in its linear mode gives good performance only for high count rate of photo-electrons, while in the case of low count rates (extremely weak signals), it must be biased above its breakdown voltage in order to have higher sensitivity. This mode of operation suffers from mean primary noise rates in the measured interval. This in turn may cause the detector stop its reaction before receiving the desired signal. When the Avalanche photodiode is biased below its breakdown voltage with highest achievable gain value, and followed by an ultra low noise amplifier, it becomes sensitive to the single photo-electron. The operation of the detector in this mode overcomes the drawbacks of the above mentioned operating mode.

\section{8. $\underline{\text { References }}$}

1- Michael E. O’Brien \& Daniel G. Fouche, "Simulation of 3D Laser Radar Systems," Lincoln Laboratory Journal, V. 15, Number 1, (2005).

2- Xiaoli Sun, Frederic M. Davidson, "Avalanche Photodiode Photon counting Receiver for Space-borne Lidar," Progress report on NAG5-356 "optical communication with semiconductor diode" for the period March 1990 - February 1991.

3- D. G. Youmans, "Avalanche photodiode detection statistics for direct detection laser radar," in Laser Radar VII: Advanced Technology for Applications, R. J. Beecherer, ed., Proc. SPIE 1633, $41-53$ (1992).

4- D.Fouche, "Detection and false alarm probabilities for laser radars that use Gieger-mode detectors," APPLIED OPTICS Vol. 42, 5388-5398 (2003).

5- Markus Henriksson "Detection probabilities for photon-counting avalanche photodiodes applied to a laser radar system," APPLIED OPTICS Vol. 44, 5140-5147 (2005).

6- George M. Williams and Andrew S. Huntington, "Probabilistic analysis of linear mode vs Geiger mode APD FPAs for advanced LADAR enabled interceptors," Proceedings of the SPIE, Vol. 6220, 622008 (2006).

7- J.W. Goodman, Statistical Optics (Wiley, New York, 1985).
8- JEFF BECK et al, "Gated IR Imaging with $128 \times 128$ $\mathrm{HgCdTe}$ Electron Avalanche Photodiode," Journal of Electronic Materials, Vol. 37, 1334-1343 (2008).

9- Meera Srinivasan; Beckett Madden-Woods; Jon Hamkins; Abhijit Biswas, "Laboratory characterization of silicon avalanche photodiodes (APD) for pulse position modulation (PPM) detection," Proc. SPIE Vol. 4272, p. 133-141, Free-Space Laser Communication Technologies XIII, (2001).

10- H. Yura, "Ladar detection statistics in the presence of pointing errors," APPLIED OPTICS, Vol. 33, No. 20 (1994).

11- Gerd Keiser , Optical Fiber Communications (McGrew-Hill, $3^{\text {rd }}$ edition,2000).

12- N.Z. Hakim, B.E.A. Saleh, M.C. Teich, "Signal to Noise Ratio for light wave systems using Avalanche Photo Diode," JOURNAL OF LIGHT WAVE TECHNOLOGY, VOL. 9, NO. 3 (1991).

13- N.Z. Hakim, B.E.A. Saleh, M.C. Teich, "Generalized excess noise factor for avalanche photodiode of arbitrary structure," IEEE Transactions on Electron Devices, Vol. 37, 599-610 (1990).

14- R. J. McIntyre, "The distribution of gains in uniformly multiplying avalanche photodiodes: Theory," IEEE Transactions on Electron Devices, Vol. 19, 703-713, (1972).

15- P. P. Webb, R. J. McIntyre, and J. Conradi, "Properties of avalanche photodiodes," RCA Review Vol. 35, 234-278, (1974).

16- M. Schwartz, "A coincidence procedure for signal detection," IEEE Trans. Inf. Theory, Vol. 2, 135-139, (1956).

17- Gregory R. Osche, "Optical Detection Theory for Laser Applications,” Pag. 383, J. Wiley, 2002.

18- A. Dorokhov, A. Glauser, Y. Musienko, C. Regenfus, S. Reucroft, J. Swain, "Recent progress on cooled avalanche photodiodes for single photon detection," Journal of modern optics, Vol. 51, 13511357(2004).

19- Khalil Seyrafi, Introduction to electro-optical imaging and tracking systems, Chapter 2, ARTECH HOUSE, INC 1993. 